This PDF is a selection from a published volume from the National Bureau of Economic Research

Volume Title: Measuring and Modeling Health Care Costs

Volume Author/Editor: Ana Aizcorbe, Colin Baker, Ernst R. Berndt, and David M. Cutler, editors

Volume Publisher: University of Chicago Press

Volume ISBNs: 978-0-226-53085-7 (cloth); 978-0-226-53099-4 (e-ISBN)

Volume URL: http://www.nber.org/books/aizc13-1

Conference Date: October 18-19, 2013

Publication Date: February 2018

Chapter Title: Measuring Output and Productivity in Private Hospitals

Chapter Author(s): Brian Chansky, Corby Garner, Ronjoy Raichoudhary

Chapter URL: http://www.nber.org/chapters/c13096

Chapter pages in book: (p. $145-172)$ 


\title{
Measuring Output and Productivity in Private Hospitals
}

\author{
Brian Chansky, Corby Garner, and Ronjoy \\ Raichoudhary
}

\subsection{Introduction}

Health care is one of the largest and most important sectors in the US economy. This sector accounted for 7.6 percent of gross domestic product (GDP) ${ }^{1}$ and 12.6 percent of all nonfarm workers ${ }^{2}$ in 2010 and continues to grow. The Bureau of Labor Statistics (BLS) projects health care and social assistance industries to generate 5.6 million new jobs by $2020,{ }^{3}$ partially in response to the growth in the population of the elderly (Henderson 2012). Within this sector, hospitals made up 28.6 percent of all employees in 2010.4 Hospitals and nursing and residential care facilities accounted for 46 percent of gross output within the health care and social assistance sector in $2010 .^{5}$

Brian Chansky, Corby Garner, and Ronjoy Raichoudhary are economists in the Office of Productivity and Technology at the US Bureau of Labor Statistics.

The authors wish to thank the Agency for Healthcare Research and Quality (AHRQ) and the Centers for Medicare \& Medicaid Services (CMS) for providing data used in this article. The views expressed here are those of the authors and do not necessarily reflect the views of the US Department of Labor or the Bureau of Labor Statistics. For acknowledgments, sources of research support, and disclosure of the authors' material financial relationships, if any, please see http://www.nber.org/chapters/c13096.ack.

1. Source: BEA, Gross-Domestic-Product-(GDP)-by-Industry Data, Value Added by Industry, Value Added as a Percentage of Gross Domestic Product (Percent); http://www.bea .gov/industry/gdpbyind_data.htm.

2. Source: Based on data from BLS, Current Employment Statistics survey; http://www.bls .gov/ces/.

3. Source: BLS, Employment Projections: Employment by Major Industry Sector; http:// www.bls.gov/emp/ep_table_201.htm.

4. Source: Based on data from BLS, Current Employment Statistics survey; http://www.bls .gov/ces/.

5. Source: Based on data from BEA, Gross-Domestic-Product-(GDP)-by-Industry Data, Gross Output by Industry, Gross Output (Millions of dollars); http://www.bea.gov/industry/ gdpbyind_data.htm. 
Over the last two decades, hospitals have experienced dramatic operational changes. Hospitals are constantly incorporating new and improved scientific advancements and technologies in an effort to improve medical care. The scale and dynamic nature of hospitals underscore the importance of measuring how efficiently hospitals are utilizing the growing labor force to provide health services.

In the United States, the concept of "health care" may be spread across many different types of activities and involve transactions in the marketplace as well as actions taken by groups and individuals. In order to study trends in the efficiency with which health services are provided, it is helpful to define the sphere where that activity takes place. A useful organization of that sphere of activity is the industry. ${ }^{6}$ Employment and hours of workers classified by industry are readily available, and by organizing economic activity along the definitions of detailed industries, the efficiency with which health services are provided by specific enterprises and distinct labor pools can be analyzed. The BLS uses this approach to produce official labor productivity measures for detailed industries. ${ }^{7}$

This chapter will analyze labor productivity specifically for private hospitals. Private hospitals comprise the nongovernment portions of two North American Industry Classification System (NAICS) industries: General Medical and Surgical Hospitals (NAICS 6221) and Specialty (except psychiatric and substance abuse) Hospitals (NAICS 6223). These hospitals employed approximately 4.6 million workers in $2010 .{ }^{8}$

Industry-specific measures of labor hours and output are required to calculate labor productivity. Measurement of labor hours for private hospitals is relatively straightforward. However, measuring output is more difficult because of the complex array of services hospitals produce. This chapter develops three models to define and measure the services provided by hospitals, and then presents the labor productivity indexes created using each model.

\subsection{What Is Hospital Output and How Can We Measure It?}

To create an output index for the hospital industry, we first must define the services provided. Next, we measure those services. Finally, we aggregate the individual services into an overall measure of output for the industry.

6. Federal data by industry is classified according to the North American Industry Classification System (NAICS). Data for this study reflect the NAICS 2007 industry definitions.

7. Bureau of Labor Statistics productivity information and data can be accessed at www.bls .gov/lpc (for labor productivity) and at www.bls.gov/mfp (for multifactor productivity).

8. Source: Based on data from the BLS, Current Employment Statistics survey; http://www .bls.gov/ces/. 


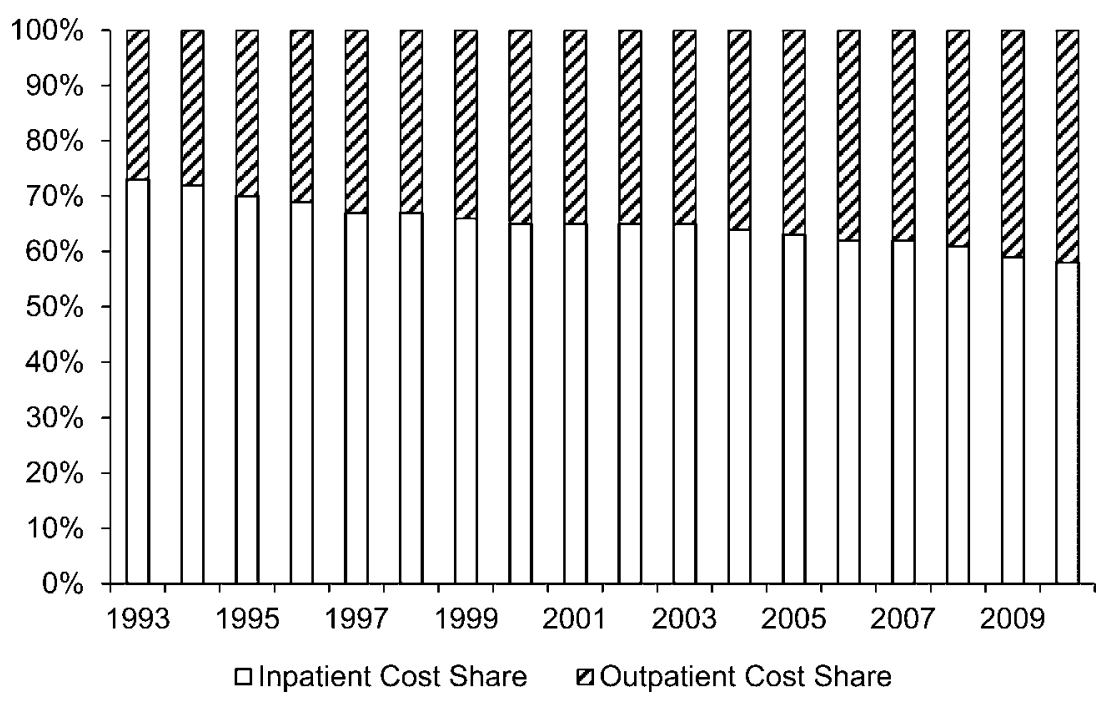

Fig. 5.1 Revenue cost shares for community hospitals, 1993-2010

We classify hospital services into two general categories, outpatient services and inpatient services. Outpatient services are those that do not require an overnight stay and can include things such as diagnostic tests or simple surgical procedures. The consumer purchases a service from the hospital, and each outpatient service can be counted as a discrete unit of output. According to the American Hospital Association (AHA), outpatient services accounted for 42 percent of total hospital revenue in 2010, up from 27 percent in 1993 (see figure 5.1). ${ }^{9}$

Inpatient services are those that require an overnight stay for extended treatment over a period of one or more days. An inpatient service requires the patient to be admitted to the hospital at the start of treatment and then to be discharged upon completion of the treatment. The course of treatment may include several different services such as diagnostic tests, surgical procedures, recuperation time, meals, and so forth. The inpatient course of treatment is designed to address a primary pathology, or reason for admission. However, secondary pathologies, often called "comorbidities," may also be treated during an inpatient stay.

The complex nature of inpatient services allows for multiple definitions of this component of output. One way to define output is as the entire bundle

9. Source: AHA, Trendwatch Chartbook 2012: Supplementary Data Table, Trends in Hospital Financing, Table 4.2: Distribution of Inpatient vs. Outpatient Revenues, 1990-2010; http:// www.aha.org/research/reports/tw/chartbook/2012/appendix4.pdf. 
of services delivered over the course of treatment during an inpatient stay (National Research Council 2010). In essence, the total course of treatment is counted as a single unit of output. This will be referred to as the course of treatment model. When a patient is admitted to a hospital, he or she is purchasing the course of treatment, which is ultimately carried out at the discretion of the hospital. In this model, the annual output of the hospital industry is based on the total number of inpatient hospital discharges and outpatient hospital visits in a given year.

Another way to define inpatient services is to disaggregate the bundle of services that comprise a course of treatment, whereby each individual service provided is counted separately. We refer to this as the procedures model. This method of defining output may be more precise, as each course of treatment may require more or less component services. Outpatient visits are treated the same under this model as the course of treatment model.

There is another aspect to defining hospital services, which is the outcome of the treatment itself. Presumably, a patient seeking treatment in a hospital wishes to have his or her pathology cured, or at least alleviated. Therefore, treatments that are unsuccessful are different in a fundamental way from those that result in a cure or alleviation. Positive outcomes of hospital treatment are the most direct indicator of the quality of service, followed by expediency and comfort.

An ideal measure of hospital output would include complete information on all of the aspects listed above. In a "perfect world" where all data were available, output would be calculated as the total number of treatments, adjusted by the number of procedures that improve outcomes. The basic service of a hospital is the course of treatment that the patient receives, so the quantity of these treatments would be defined as output. To account for the quality of the services provided, the quantity of treatments would be adjusted based upon how successful they were. However, to ensure that the outcome of the treatment was attributable to the quality of hospital care, each individual procedure would be evaluated to determine how much utility was provided.

Unfortunately, the measurement of treatment outcomes is not feasible, mainly due to a lack of data availability. Currently, the only available patient-level statistic related to outcomes is whether an inpatient died during the course of treatment. However, this mortality statistic does not provide a complete story, since the health status of patients that did not die is unknown. Even if data were available on the exact number of patients that were not cured, a true measure of outcomes would require knowledge of the role the hospital's care plays in these outcomes. This is a difficult task due to the many exogenous factors that can play a role in the recovery of a patient. Factors such as diet, lifestyle, genetics, and even random chance can affect the success of specific treatments. Therefore, due to the complexities involved in the measurement of outcomes, the measures of 
hospital output presented in this chapter do not account for the outcome of treatments. $^{10}$

Two primary methods to measure industry output are physical quantity and deflated value. Both the course of treatment and procedures models of hospital output are based on physical quantities of inpatient and outpatient services. The deflated value method measures output based on revenues that have been adjusted with one or more price indexes to remove the effect of price change. With this method, an industry's deflated revenue serves as a proxy for the quantity of output. We refer to this approach as the revenue model.

Like most service industries, the hospital industry provides many different types of services. We combine these services into a single index of output using weights based on the value of each service relative to the total value of output. Thus, the effect of each individual service on the change in total output is proportional to the amount of resources required to provide that service. ${ }^{11}$

\subsection{Data Sources}

To create the three models of hospital output and subsequent indexes of labor productivity, we use the following data sources:

BLS Industry Employment and Hours Data. The measure of labor hours used to calculate labor productivity come from the BLS industry productivity program's comprehensive database of employment and hours. ${ }^{12}$ This labor index represents the sum of hours of all workers in private hospitals each year. This measure is based primarily on data from the BLS Current Employment Statistics program, supplemented with data from the Current Population Survey. Annual hours are estimated separately for General Medical \& Surgical Hospitals (NAICS 6221) and Specialty Hospitals (NAICS 6223) and then summed.

Nationwide Inpatient Sample (NIS). Our measures of inpatient services are based on patient discharge data from the NIS. The NIS is the largest all-payer inpatient care database publicly available in the United States,

10. A number of other countries' statistical agencies have investigated methods and developed measures for quality change to adjust their health statistics. As of the publication of this chapter, no single method of measuring quality change has been widely adopted internationally. For a discussion of potential quality-change measurements, see Douglas $(2006,13)$. For a discussion of the reasons quality-change metrics were not ultimately included, see Statistics New Zealand $(2013,8)$.

11. The Industry Productivity Program releases labor productivity measures that incorporate annual chain-weighted indexes for measuring changes in industry output. The Tornqvist index aggregates the growth rates of various industry outputs with annual weights based on the products' shares in total value of industry production. For a more detailed look at Tornqvist aggregation, see the BLS Handbook of Methods, chapter 11: "Industry Productivity Measures."

12. Bureau of Labor Statistics, Labor Productivity and Costs, Industry Employment and Hours Data Tables; http://www.bls.gov/lpc/iprhoursdata.htm. 
providing information on health care utilization and costs. The unit of observation is an inpatient stay record. The NIS contains discharge-level records, rather than patient-level records. Therefore, individual patients who are hospitalized more than once in one year may be present in the NIS multiple times. We use the NIS to count all inpatient discharges and procedures in private hospitals for each year from 1993 to $2010 .{ }^{13}$ Each discharge is assigned a diagnosis-related group (DRG) that corresponds to both the primary pathology being treated as well as the associated bundle of procedures and services used during treatment. A single charge is reported for the complete stay.

American Hospital Association ( AHA) Annual Survey. Data for the quantity of outpatient visits comes from the AHA Annual Survey. An outpatient visit is defined as a:

Visit by a patient not lodged in the hospital while receiving medical, dental, or other services. Each visit an outpatient makes to a discrete unit constitutes one visit regardless of the number of diagnostic and/or therapeutic treatments that the patient receives. Total outpatient visits should include all clinic visits, referred visits, observation services, outpatient surgeries, and emergency room visits. ${ }^{14}$

National Hospital Ambulatory Medical Care Survey (NHAMCS). While the AHA Annual Survey includes extensive data on the quantity of outpatient visits, the survey does not provide detail regarding diagnosis. The NHAMCS, however, does provide a measure of outpatient visits separated into major disease categories. ${ }^{15}$ The ratios of each major disease category are applied to total AHA outpatient visits.

CMS Statistical Supplement. The Medicare and Medicaid Statistical Supplement provides comprehensive annual data about Medicare, Medicaid, and other CMS programs. The CMS compiles billing data for hospital outpatient visits that are covered by Medicare and Medicaid, including the total number of outpatient visits and the associated charges for each of the 900-plus ICD-9-CM ${ }^{16}$ categories. The total visits and charges for individual ICD-9-CM categories are aggregated to the level of sixteen major disease category groupings.

13. The NIS is built from data provided by state health agencies. Prior to 1993 , only a small number of states were sampled. Thus, based primarily on the addition of states to the NIS data set over time, HHS recommends that time series analyses on these data begin with 1993.

14. Source: AHA, Trendwatch Chartbook 2009: Trends Affecting Hospitals and Health Systems, Glossary; http://www.aha.org/research/reports/tw/chartbook/2009/glossary.pdf.

15. The scope of the AHA data set is greater than that of the NHAMCS. The AHA collects data from 98 percent of community hospitals, making it the preferred source of outpatient data.

16. The International Classification of Diseases, Ninth Revision, Clinical Modification (ICD-9-CM) is the official system of assigning codes to diagnoses and procedures associated with hospital utilization in the United States; http://www.cdc.gov/nchs/icd/icd $9 \mathrm{~cm} . \mathrm{htm}$. 
Table 5.1

Data sources for output

\begin{tabular}{|c|c|c|}
\hline Data source & Provider & Element \\
\hline \multicolumn{3}{|c|}{ Course of treatment and procedures models: Inpatient } \\
\hline & & Number of discharges per DRG \\
\hline NIS & HCUP & Number of procedures per DRG \\
\hline & & Cost per DRG discharge \\
\hline \multicolumn{3}{|c|}{ Course of treatment and procedures models: Outpatient } \\
\hline AHA Annual Survey & AHA & $\begin{array}{l}\text { Total number of outpatient visits } \\
\text { Inpatient and outpatient revenue shares }\end{array}$ \\
\hline \multicolumn{3}{|c|}{ Revenue model } \\
\hline SAS & Census & Total revenue for community hospitals \\
\hline PPI & BLS & Industry price deflators \\
\hline
\end{tabular}

Service Annual Survey $(S A S)$. The primary source for revenue data is the SAS from the Census Bureau, which publishes total hospital revenue by industry for General Medical and Surgical Hospitals (NAICS 6221) and for Specialty (except psychiatric and substance abuse) Hospitals (NAICS 6223). The aggregate value includes revenues for inpatient and outpatient services, plus miscellaneous services such as food and parking revenue.

Producer Price Index (PPI). Total revenue for each industry is deflated using appropriate BLS PPIs. ${ }^{17}$ The PPIs for general and specialty hospitals define output as a bundle of services provided for the treatment of a medical condition, not as the individual services rendered such as $\mathrm{x}$-rays, drugs, and medical supplies (Catron and Murphy 1996).

Table 5.1 provides a brief summary of the data sources used in each model of output. More information about these data sources can be found in appendix A.

\subsection{Three Models of Output}

\subsubsection{The Course of Treatment Model of Output}

The course of treatment model defines hospital output as the full course of treatment received by a patient admitted to a hospital. This model is based on the "direct quantity index" approach suggested by Triplett (2012). The final output index is an aggregation of inpatient and outpatient services.

\section{Inpatient Stays}

The physical quantity measure of output for inpatient stays is a weighted aggregation of patient discharge data from the NIS. The total number of

17. NAICS 6221 is deflated using PPI 622110622110 G, and NAICS 6223 is deflated using PPI $6223106223106 \mathrm{G}$. 
inpatient discharges in each DRG category are combined into a single quantity index of inpatient output with weights based on each DRG's share of total cost. Equation (1):

$$
\frac{Q_{t}}{Q_{t-1}}=\exp \left[\sum_{i=1}^{n} w_{i, t}\left(\ln \left(\frac{q_{i, t}}{q_{i, t-1}}\right)\right)\right],
$$

where

$Q_{t} / Q_{t-1}=$ the ratio of inpatient output in the current year $(t)$ to the previous year $(t-1)$, $n=$ the number of DRGs, $\ln \left(q_{i, t} / q_{i, t-1}\right)=$ the natural logarithm of the ratio of the quantity of inpatient

stays for DRG $i$ in the current year to the quantity in the previous year, and $w_{i, t}=$ the average value share weight for DRG $i_{t}$ and DRG $i_{t-1}$.

Total inpatient values are calculated by multiplying the quantity of inpatient treatments for each DRG category by their associated average cost or average charge. Relative weights based on these values are derived by dividing the total value for each DRG category in a given year by the sum of values for all DRGs in that year.

We use average cost data for 2001 forward. Cost is defined as the dollar amount incurred by a hospital to provide services. Prior to 2001, cost data are unavailable, so we use average charge data for that period. Charge is defined as the final amount on a patient's bill.

Costs are a more accurate measure of the value of an inpatient stay because they are not subject to exogenous price factors. In those years where cost data are unavailable, the charge data are an acceptable proxy because the primary factor that determines the relative charge for inpatient services is the value of hospital resources used in the provision of such services.

\section{Outpatient Services}

We use the number of outpatient visits from the AHA Chartbook as the basis of our physical quantity measure of outpatient services. ${ }^{18}$ To obtain a more accurate representation of outpatient output, we disaggregate the yearly outpatient totals from AHA into sixteen major disease categories using data from the NHAMCS.

We match the number of visits for each major disease category with corresponding charge data from CMS. The total charges are divided by the number of visits for each respective major disease category to obtain an average charge per outpatient visit. We then multiply average charges by the quantity of outpatient visits that are calculated using data from AHA

18. American Hospital Association statistics do not separate private from government hospitals. The NIS data is used to calculate a percentage of hospitals that are privately owned in the United States. This ratio is applied to the AHA data to remove government-owned hospitals. 
and CDC. The resulting values are used to derive weights for combining the quantities of various outpatient visits. ${ }^{19}$

A variation of equation (1) is used to calculate outpatient index growth, where

$Q_{t} / Q_{t-1}=$ the ratio of outpatient output in the current year $(t)$ to the previous year $(t-1)$,

$n=$ the number of major disease categories,

$\ln \left(q_{i, t} / q_{i, t-1}\right)=$ the natural logarithm of the ratio of the quantity of outpatient

visits for major disease category $i$ in the current year to the quantity in the previous year, and $w_{i, t}=$ the average value share weight for MDC $i_{t}$ and $\operatorname{MDC} i_{t-1}$.

It is possible that the use of Medicare and Medicaid charges may introduce some bias to the outpatient measure, as these charges are drawn from only a subset of the population. However, as with the charge data used to weight the inpatient services, the value of hospital resources used in order to provide the outpatient services is thought to be the primary factor affecting these charges. Therefore, we expect shares for each major disease category calculated from the CMS data to be similar to corresponding shares for the general population. The various quantities of outpatient visits are combined into a single outpatient index using their associated share of the total nationwide value as weights.

An alternative data source for outpatient visits and charges is the Medical Expenditure Panel Survey (MEPS). See appendix B for an outpatient index created using these data.

\section{Output Index}

We combine the independently constructed indexes of inpatient services and outpatient services to create an output index for private hospitals using the course of treatment model. Percentages of inpatient and outpatient gross revenue from AHA's Trendwatch report ${ }^{20}$ are used to calculate the average share weights for inpatient and outpatient services. Equation (2):

$$
\ln \left(\frac{A_{t}}{A_{t-1}}\right)=w_{i}\left(\ln \frac{I_{t}}{I_{t-1}}\right)+w_{o}\left(\ln \frac{O_{t}}{O_{t-1}}\right)
$$

where

$A=$ output index for community hospitals,

$I=$ Inpatient input,

19. The CMS data are only available from 2004 to the present. Therefore, all average charge data are held constant from 1993 to 2003, using the 2004 values.

20. American Hospital Association, Trendwatch Chartbook 2012: Supplementary Data Table, Trends in Hospital Financing, Table 4.2: Distribution of Inpatient vs. Outpatient Revenues, 1990-2010; http://www.aha.org/research/reports/tw/chartbook/2012/appendix4.pdf. 
$O=$ Outpatient input, and

$w_{i}, w_{o}=$ value share weights.

\subsubsection{Review of the Course of Treatment Model}

The course of treatment model has several strengths. The data on inpatient discharges and outpatient visits are comprehensive and reliable. A wealth of information about the activity of hospitals in the United States has accumulated over time from a number of sources including NIS, NHAMCS, AHA, and CMS. These data sources can be used to calculate the total number of courses of treatment and their associated charges/costs for the entirety of US private hospitals. Also, the course of treatment model of output is sensible from a demand perspective. Whether a patient goes to a hospital for a few hours or a few days, the objective is the same: to have a particular health problem treated. The idea that hospital output should be defined as a course of treatment has been advocated by a number of health economists (Triplett 2012).

The simplicity of a course of treatment measure of output may also be the model's primary weakness, however. As opposed to the ideal "perfect world" scenario described earlier in this chapter, the course of treatment measure lacks the robustness of data that could be provided by including procedure and outcome data. A count of hospital discharges does not provide as much information about the quantity of individual health services delivered over time as does a count of the actual procedures provided during the hospital stay. Instead, in the course of treatment model different output intensities related to each DRG are implicitly accounted for in the average value share weights used to combine the various DRG categories of a hospital stay.

A measure of output based on courses of treatment also does not address issues relating to variable outcomes and changes in the quality of treatment over time. When treatments improve over time, ideally they should be counted as an increase in output. A disadvantage of a physical quantity measure of output is that it cannot directly account for quality change. Instead, all courses of treatment are counted equally, regardless of the result. While it can be argued that unsuccessful treatments should not be counted as equal output, the data to accommodate this distinction are not currently available. Although there are numerous sources that measure some aspects of variable outcomes and quality change in the health services sector, there is no broad agreement on how to apply this type of data to nationwide statistics. ${ }^{21}$ Until a consensus is reached on this topic, a measure free of these complicating factors is a more sensible approach for the measurement of hospitals' output. ${ }^{22}$ Given the current data limitations, the simplicity and directness of the course

21. We incorporate a mortality data element into the course of treatment inpatient model to create a survival-adjusted series (see appendix C).

22. The output measures for service-providing industries currently maintained by DIPS are not adjusted for variable outcomes, although this issue may be present to a lesser degree. Furthermore, when a physical-quantity approach is used, quality changes are not accounted for. 
of treatments model may actually be an advantage. By using only data that are highly reliable, the resulting output index can be assumed trustworthy.

Comorbidities, which are defined as one or more disorders that are present in addition to a primary pathology, also pose an obstacle to the accurate measurement of hospital services. Ideally, when a patient is treated for multiple disorders, each pathology should be accounted for individually. Unfortunately, the level of data needed to separate each comorbidity into its own category of treatment is not available. The DRG system has recognized this problem, however, and many diagnosis groups have been split to account for cases where there are serious complications or comorbidities. Presumably these DRGs, which include complications and comorbidities, have higher average charges/costs and thus contribute more weight to the overall inpatient index. By organizing hospital discharges according to the DRG system, the course of treatment model of output indirectly accounts for the presence of comorbidities in private hospitals.

\subsubsection{The Procedures Model of Output}

As discussed previously, hospital output can be defined as a procedure rather than a course of treatment. The NIS provides some data that can be used to measure these detailed services. Specifically, for each hospital inpatient stay the NIS records the number and types of procedures performed. A procedure is defined as a medical intervention that was performed during a hospital stay. Procedures are classified into one of four broad categories: minor diagnostic, minor therapeutic, major diagnostic, and major therapeutic. Examples of common procedures include blood transfusions, cesarean sections, vaccinations, and kidney dialysis. According to the Agency for Healthcare Research and Quality (AHRQ), in 2007 approximately 70 percent of hospital inpatient stays included at least one procedure (Stranges, Russo, and Friedman 2009). We use a physical quantity approach based on the number of procedures per DRG to develop an alternative measure of inpatient output of the hospital industry.

While charge or cost data are available for total inpatient stays, values for individual procedures are not available in the NIS. This precludes an accurate valuation of procedures across different DRGs. A reasonable alternative is to count the total yearly number of procedures within each DRG, and then weight them together using the associated DRG charge/cost data. This method uses the number of procedures as the unit of output within the context of the DRG classification system. Equation (3):

$$
\frac{Q_{t}}{Q_{t-1}}=\exp \left[\sum_{i=1}^{n} w_{i, t}\left(\ln \left(\frac{q_{i, t}}{q_{i, t-1}}\right)\right)\right]
$$

where

$Q_{t} / Q_{t-1}=$ the ratio of inpatient output in the current year $(t)$ to the previous year $(t-1)$, 
$n=$ the number of DRGs

$\ln \left(q_{i, t} / q_{i, t-1}\right)=$ the natural logarithm of the ratio of the quantity of procedures

in DRG $i$ in the current year to the quantity in the previous year, and $w_{i, t}=$ the average value share weight for DRG $i_{t}$ and DRG $i_{t-1}$.

As with the course of treatment model, the procedures index of inpatient output is combined with an index of outpatient services. The outpatient index for the procedures model is identical to that of the course of treatment model (see equation [2]). Because an outpatient visit is defined as a single encounter in a hospital rather than a bundle of services, the count of procedures and treatments is the same. The purpose of an outpatient visit can be considered as either a single procedure or a single treatment.

\subsubsection{Review of the Procedures Model}

A procedures model provides more detail about actual health services provided during inpatient stays than does a simple count of admissions or discharges. In this model, increases in the average number of procedures performed per inpatient stay will lead to growth in the output index. When output of a hospital is defined as individual services performed, a procedures model of output may be preferable. Additional procedures may result in improved diagnosis or treatment of pathologies that benefit the patient. This benefit would be missed with other methods such as the courses of treatment measure of output. However, it is not clear that all procedures that are undertaken are necessary or that an increase in the number of procedures necessarily leads to better treatment of a pathology. While the procedures model may be attractive from the hospital's viewpoint, to define procedures as the hospital's unit of output can create a conflict with the needs of the patient. Data currently available are not sufficient to construct a direct link between the number of procedures provided by hospitals and the outcomes of treatments. Patients seek to have their pathology addressed, and yet the precise type and number of procedures is generally left to the hospital's discretion. From the point of view of the consumer, additional procedures do not necessarily represent an increase or improvement in the health service received.

Other than a mortality variable (whether the patient was discharged alive or dead), there are no data elements currently available that provide information on the efficacy of the course of treatment. And even if such data were available, it would be difficult to ascertain whether the increased number of procedures were the cause of improved outcomes, or if the improvements were due to factors outside the hospital's control. One could conceive of situations where an increase in the number of procedures even has a negative effect on a patient's health, such as a misdiagnosis or treating an infection acquired during a hospital stay. More accurate data on the link between procedures and treatment outcomes would be useful. There are other problems with the procedures approach for an inpatient index. Some hospital 
inpatient stays do not require any procedures as classified by ICD-9-CM. For example, inpatient stays for the sole purposes of bed rest or observation fall into this category. The DRGs in which these types of stays are common would likely be underrepresented in a procedures inpatient index. Also, the lack of charge/cost data for different procedures prevents the use of weights that would accurately adjust the inpatient index based on changes in the mix of procedures over time.

\subsubsection{Output Derived from Revenue}

The majority of industry productivity measures produced by the BLS use a deflated value concept of output measurement. In these industries, deflated revenues serve as a proxy for quantities of goods and services produced. Deflated value methodology is used because in most cases, industry revenue data are more available than physical quantity data. Ideally, revenue data should be as detailed as possible to account for the variety of different services provided by hospitals and each revenue category should be deflated with a price deflator specific to that category. The SAS reports total hospital revenue; however, it is not disaggregated into detailed services provided. Instead, total revenue for each industry is deflated using the BLS PPIs for the industry. ${ }^{23}$ The final output index produced with this model is an aggregation of the deflated revenues for NAICS 6221 and NAICS 6223. Due to a lack of data availability, inpatient and outpatient services are not calculated independently under the revenue model, as is the case with the course of treatment and procedures models.

\subsubsection{Review of the Revenue Model}

An important benefit of a revenue model is the potential to account for quality change through the use of price deflators. Price deflators that are adjusted for quality change ensure that revenue increases that are driven by inflation are removed from the output series, while those that are driven by quality improvements are not. To the extent that quality adjustments are made to the price indexes, a deflated value measure of output allows for an increase in the actual services rendered by a hospital to be counted (Aizcorbe and Nestoriak 2008). The general medical and surgical hospital PPI currently accounts for quality change by incorporating quality indicators for three DRGs: heart attack, heart failure, and pneumonia (Hospital Quality Valuation Team 2008).

Some health economists have cautioned that current price indexes may not adequately respond to changing treatments. For example, Aizcorbe and Nestoriak (2011) suggest that use of a standard fixed-basket index inflates price growth. They suggest a medical care expenditure index to address this

23. NAICS 6221 is deflated using PPI 622110622110 G, and NAICS 6223 is deflated using PPI $6223106223106 \mathrm{G}$. 
concern. These types of price indexes "track the overall cost of care (all expenditures), not the cost of the individual services." At present, medical care expenditure indexes specific to hospitals are not available. However, the BLS has recently developed hospital PPIs on a disease basis. Beginning in 2008, PPIs are available for major diagnostic categories (MDC) within General Medical and Surgical Hospitals. These indexes represent price change for all hospital services and are grouped according to the system of the body being treated (e.g., circulatory system, digestive system, etc.). All payer types (e.g., Medicare, Medicaid, private insurance, etc.) are covered by these price deflators. ${ }^{24}$

The quinquennial Economic Census of 2007 is the first to report detailed hospital revenue based on both MDCs as well as ancillary hospital services. If this detailed data continues with the 2012 Economic Census, it could potentially be combined with the BLS MDC-based PPI to create an improved deflated-value measure of output.

There are several characteristics of the hospital industry that present challenges to measuring output based on revenues and prices. First, there are a variety of types of payers for hospital services, and hospital prices are not uniform for all customers. Different patients pay different amounts for the same hospital services based on whether payment is out of pocket, through private insurance, or Medicare/Medicaid. Because prices are negotiated between insurers and hospitals, the amount of revenue received for hospital services varies from patient to patient. The assumption that prices reflect marginal costs does not necessarily hold in the hospital industry because prices are administered rather than reached through open competition. Cylus and Dickensheets $(2007,61)$ recognize that "it can be argued that deriving outputs using nominal payments and the hospital PPI results in a distorted measure."

Another unique characteristic of the hospital industry is that the provider (e.g., physician) often chooses the services provided. This is because the consumer may be a minor, unconscious, lacking knowledge of medical procedures, or simply apathetic because the payments are made by third-party insurance plans. Rosen and Cutler $(2007,54)$ point out that "In medical care, however, the link between purchase and value is not clear ... [Thus], most health care analysts do not assume that purchase decisions will reflect the true value of the good." In addition, different hospitals may choose to treat the same pathologies in different ways, and the revenue they receive from these courses of treatment will reflect such choices. Thus, the revenue from any given course of treatment differs from hospital to hospital, based on decisions made by the hospitals themselves.

24. The MDC-based PPIs are actually available going back to 1992. However, these older PPIs only cover private insurance patients. They do not account for bills paid with Medicare, Medicaid, or other public sources, which combined in 1997 for 62 percent of all hospital gross patient revenues (Lane 2001). 


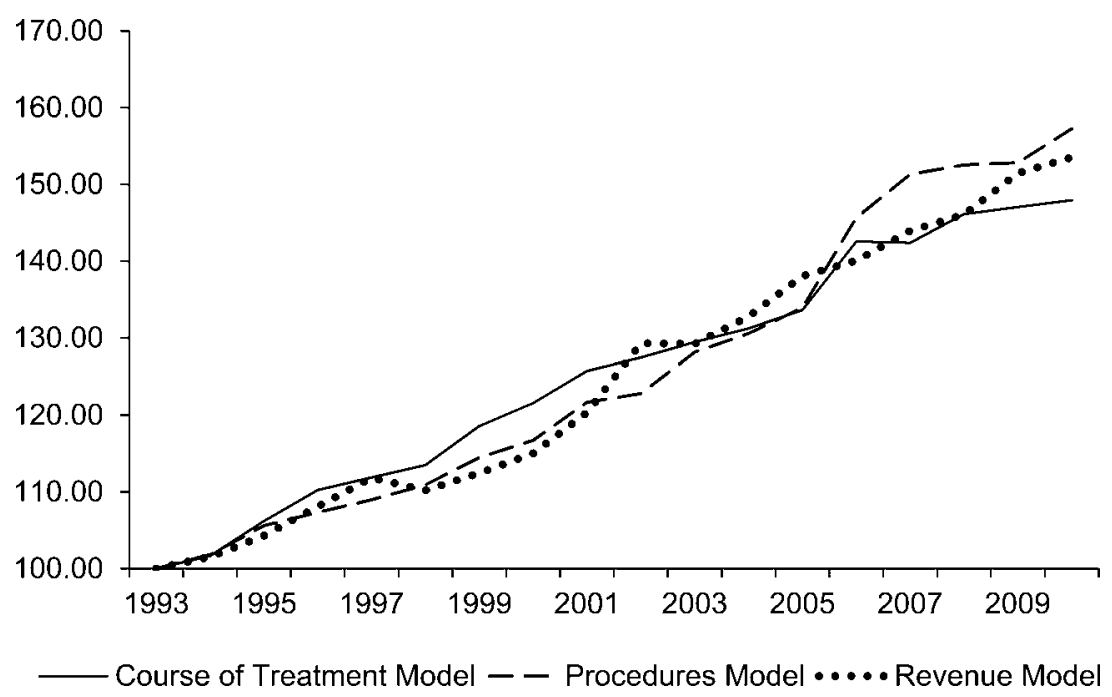

Fig. 5.2 Output for private hospitals, 1993-2010

Deflated values serve as a proxy for quantity, and are of interest in assessing trends in hospital output. However, in the hospital industry, the data sources for physical quantity are more accurate and more comprehensive than those for revenue. Furthermore, there are industry-specific factors, such as the complex structures of payments, which can cause revenues to move differently than services. For these reasons, we prefer physical quantities to deflated revenues.

\subsection{Results}

Over the last two decades, hospitals have experienced dramatic changes in the way they operate. As with any industry that experiences significant technological change, labor productivity is expected to increase. All three models of output show positive growth for output and labor productivity for 1993-2010. However, labor productivity varies in the selected subperiods (see figures 5.2 and 5.3).

\subsubsection{Course of Treatment Model}

With the course of treatment model of output, real output of private hospitals exhibits average annual growth of 2.3 percent from 1993 to 2010 and shows positive year-to-year growth every year except in 2007. The indexes of inpatient and outpatient services posted yearly average growth rates of 1.7 and 3.5 percent, respectively, from 1993 to 2010 (see figure 5.4). The hospital industry experienced long-term average annual labor productivity growth of 0.7 percent from 1993 to 2010 (see table 5.2). 


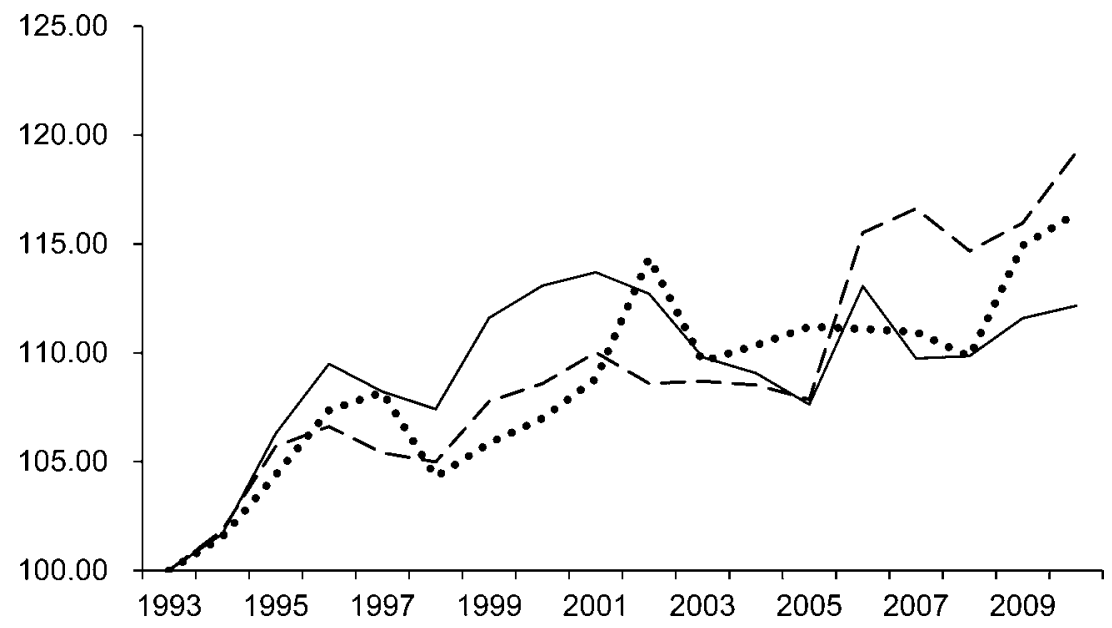

— Course of Treatment Model - - Procedures Model $\cdots . .$. Revenue Model

Fig. 5.3 Labor productivity for private hospitals, 1993-2010

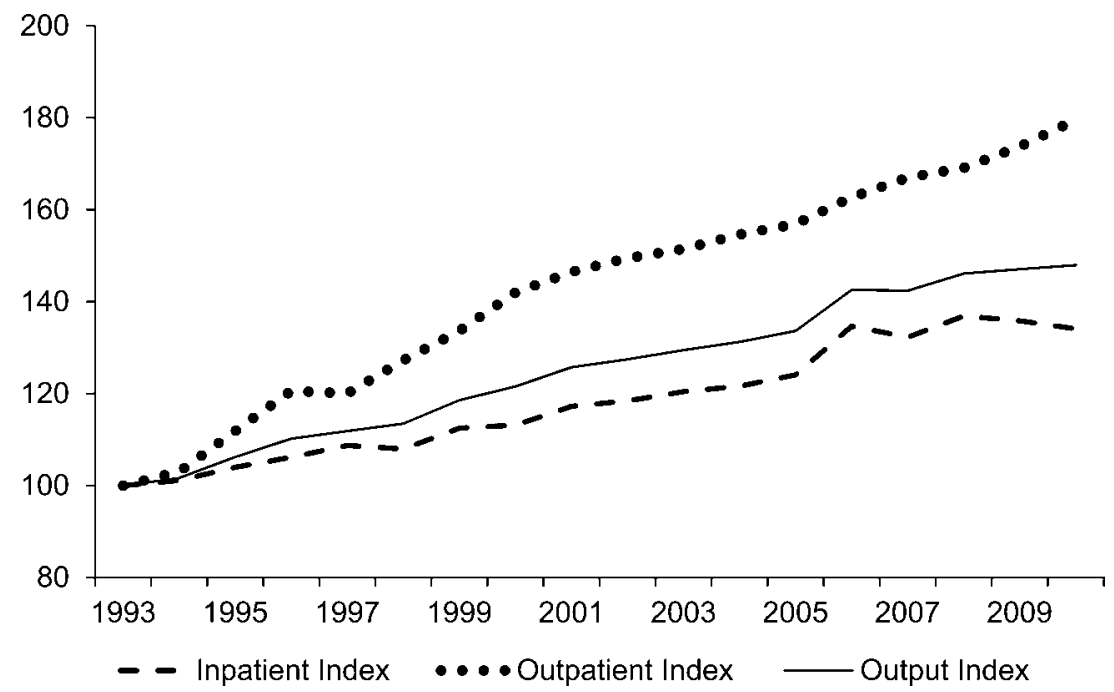

Fig. 5.4 Indexes of inpatient services, outpatient services, and combined output for community hospitals, course of treatment model: 1993-2010 
Table 5.2

Average annual percent change in labor productivity for private hospitals

\begin{tabular}{lccc}
\hline Time period & $\begin{array}{c}\text { Course of treatment model } \\
(\%)\end{array}$ & $\begin{array}{c}\text { Procedures model } \\
(\%)\end{array}$ & $\begin{array}{c}\text { Revenue model } \\
(\%)\end{array}$ \\
\hline $1993-2010$ & 0.7 & 1.0 & 0.9 \\
$1993-2001$ & 1.6 & 1.2 & 1.1 \\
$2001-2010$ & -0.2 & 0.9 & 0.8 \\
\hline
\end{tabular}

Table 5.3

Average annual percent change in labor productivity and related series for private hospitals using a course of treatment model of output, 1993-2010 and selected subperiods

\begin{tabular}{lccc}
\hline & $\begin{array}{c}1993-2010 \\
(\%)\end{array}$ & $\begin{array}{c}1993-2001 \\
(\%)\end{array}$ & $\begin{array}{c}2001-2010 \\
(\%)\end{array}$ \\
\hline Output index & 2.3 & 2.9 & 1.8 \\
Inpatient index & 1.7 & 2.0 & 1.5 \\
Outpatient index & 3.5 & 4.9 & 2.3 \\
Hours index & 1.6 & 1.3 & 2.0 \\
Labor productivity & 0.7 & 1.6 & -0.2 \\
\hline
\end{tabular}

Labor productivity grew on average by 1.6 percent per year for the years 1993-2001. In contrast, growth in labor hours outpaced that of output from 2001 through 2010 , leading to an average annual decline in labor productivity of 0.2 percent for the time period (see table 5.3).

The decrease in productivity for the years 2001-2010 may seem to contradict conventional wisdom, but is not totally unexpected. As technology in the medical field advances, procedures that once required an inpatient stay can now be performed on an outpatient basis inside or outside the hospital. As a result, the remaining inpatient cases being treated by hospitals have become increasingly difficult and complex. These types of treatments would likely require more staff attention, and thus more hours. This is one explanation for the greater growth in labor hours relative to output for 2001-2010.

\subsubsection{Procedures Model}

With the procedures model, output experienced long-term average growth of 2.7 percent per year for the period 1993-2010. As figure 5.5 shows, the outpatient index grows at a faster rate than the inpatient index. As with the course of treatment model, however, the final output index is influenced more heavily by the change in the inpatient index because the inpatient services require more resources and therefore have a larger weight. Labor productivity rose at a long-term annual rate of 1 percent for the period 1993-2010 (see table 5.2). 


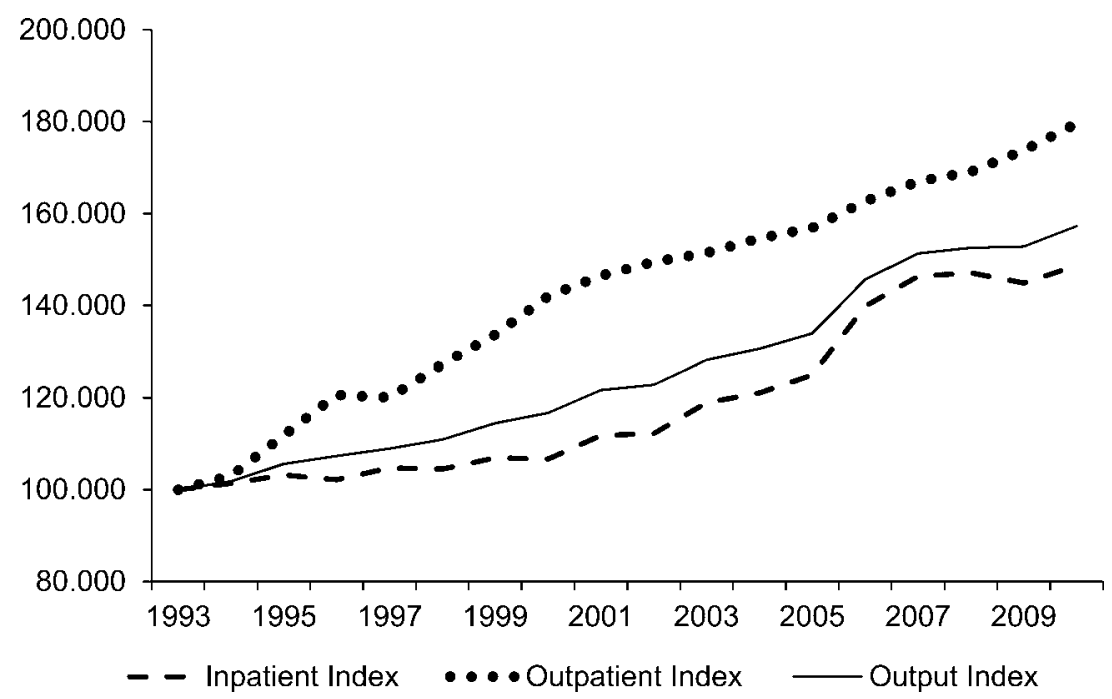

Fig. 5.5 Indexes of inpatient services, outpatient services, and output for community hospitals, procedures model: 1993-2010

\subsubsection{Revenue Model}

The long-term yearly average growth of output under the revenue model is 2.6 percent for the period 1993-2010. The same labor index from the previous two models is used, leading to a 0.9 percent average annual gain in labor productivity during the period.

\subsection{Conclusion}

Each of the three output models discussed in this chapter has strengths and weaknesses. For a model to be broadly accepted there are two concerns to be addressed: the data must be accurate and the definition of output must be compelling. The revenue model presented in this chapter is weak in both the availability of the data and the definition of output. Revenue and price data are not yet detailed enough to achieve in-depth coverage of the hospital industry. Additionally, the weak link between consumer choices, services rendered, and compensation received implies a tenuous relationship between revenue and actual output. The promise of the deflated value method is the ability to more easily incorporate quality change through prices. More research into these issues would be needed for a revenue model to be considered.

Of the two physical quantity models, the course of treatment model has the advantage of more precise data. Inpatient treatments are categorized by DRG, a common practice in the hospital industry, and each inpatient treatment is weighted using matching charge/cost data. This is more reliable than the pro- 
cedures model, where the data are lacking to organize and value the different types of procedures. Although the course of treatment model does not explicitly account for hospital quality change, a non-quality-adjusted measure can be beneficial as a baseline against which to judge future work in this field.

The ultimate question of how to define output in the hospital industry is subjective and open to debate. However, in our opinion, the most natural way to define the output of an industry is to answer the question: What services are being demanded? For hospitals, the consumer is purchasing the service of treatment for a specific health problem. We believe counting the full courses of treatments has the advantage of data availability, and is the most direct way to determine industry output.

\section{Appendix A}

\section{Data Sources in Depth}

Befitting such a vast and complex part of the economy, there are a number of different government agencies and private organizations that measure the activity of the health care sector. The US Department of Health and Human Services (HHS) administers a wide variety of data-collection programs covering the nation's health care infrastructure. These include the Nationwide Inpatient Sample (NIS) and the National Hospital Discharge Survey (NHDS), which collect information on hospital inpatient care. Data on physicians' offices and emergency departments are provided by the National Ambulatory Medical Care Survey (NAMCS) and the Nationwide Emergency Department Sample (NEDS), respectively. The Medical Expenditure Panel Survey (MEPS) provides data on the cost and use of health care and health insurance coverage. Additionally, the Centers for Medicare and Medicaid Services (CMS) collects a wealth of data relating to government-sponsored health insurance programs.

Outside of HHS, the Census Bureau collects revenue data for the health care sector in its Services Annual Survey (SAS) and the quinquennial Economic Census, while the Bureau of Economic Analysis (BEA) measures the economic activity of the health care sector in its National Income Product Accounts (NIPAs). In the private sector, the American Hospital Association (AHA) collects a wealth of data on its members, which number over 6,500 hospitals.

We use the following data sources to create our models of hospital output:

Nationwide Inpatient Sample (NIS). Our measures of inpatient services are based on patient discharge data from the NIS. Sponsored by the Agency for Healthcare Research and Quality (AHRQ), the NIS is the largest all-payer inpatient care database publicly available in the United States, providing 
information on health care utilization and charge data, with annual data starting in 1988. As part of the Healthcare Cost and Utilization Project (HCUP), the NIS is drawn from those states participating in HCUP; for 2010, these states comprise over 96 percent of the US population. The 2010 database contains information on approximately eight million hospital stays from 1,051 hospitals in forty-five states sampled to approximate a 20 percent stratified sample of US community hospitals. The NIS is a stratified probability sample of hospitals in the frame, with sampling probabilities proportional to the number of US community hospitals in each stratum. The universe of US community hospitals is divided into strata using five hospital characteristics: ownership/control, bed size, teaching status, urban/ rural location, and US region.

The unit of observation is an inpatient stay record. Inpatient stay records in the NIS include clinical and resource use information typically available from discharge abstracts. It includes more than 100 data elements for each hospital stay, including primary and secondary diagnoses, admission and discharge status, hospital characteristics, expected payment source, primary and secondary procedures, length of stay, patient demographics, and total costs/charges. ${ }^{25}$ The NIS is the only national hospital database with charge information on all patients, regardless of payer, including persons covered by Medicare, Medicaid, private insurance, and the uninsured ${ }^{26}$ The NIS contains discharge-level records, rather than patient-level records. Therefore, individual patients who are hospitalized more than once in one year may be present in the NIS multiple times.

We use the NIS to count all inpatient discharges in private hospitals for each year from 1993 to $2010 .{ }^{27}$ Each discharge is assigned a diagnosis-related group (DRG) that corresponds to both the primary pathology being treated as well as the associated bundle of procedures and services used during treatment. These treatment bundles correspond to differing amounts of hospital resource utilization. The DRG classification system was developed and is used by the Centers for Medicare and Medicaid Services (CMS) to create a uniform payment system for Medicare and Medicaid patients across the United States. ${ }^{28}$ The DRGs are the primary means by which

25. Healthcare Cost and Utilization Project, Overview of the Nationwide Inpatient Sample (NIS); http://www.hcup-us.ahrq.gov/nisoverview.jsp.

26. Healthcare Cost and Utilization Project, Introduction to the HCUP Nationwide Inpatient Sample (NIS) 2009, Abstract; http://www.hcup-us.ahrq.gov/db/nation/nis/NIS_2009 _INTRODUCTION.pdf.

27. The NIS is built from data provided by state health agencies. Prior to 1993, only a small number of states were sampled. Thus, based primarily on the addition of states to the NIS data set over time, HHS recommends that time series analyses on these data begin with 1993.

28. The DRGs are derived from the International Classification of Diseases, Ninth Revision, Clinical Modification (ICD-9-CM) (US Department of Health and Human Services, Centers for Medicare and Medicaid Services). Official version: International Classification of Diseases, Ninth Revision, Clinical Modification, Sixth Edition. DHHS Pub No. (PHS) 06-1260. 
hospital discharges are categorized nationwide. They constitute a reasonable way to categorize inpatient services for the purpose of constructing an inpatient index because each patient is assigned a single DRG (as opposed to a set of multiple procedures or diagnoses) with one corresponding charge. The classification of diagnoses in the DRG system are updated annually to include more specific types of ailments as well as differing levels of severity. New DRGs are also assigned for substantially new methods of treatment. ${ }^{29}$

For the purposes of this study, we remove state and local government hospitals from the original NIS data set, leaving only the privately owned nonprofit and for-profit hospitals. We do this to ensure consistency between the output measure and the BLS labor input series that is used in the final labor productivity calculations. ${ }^{30}$ The sample of discharge records is made into a nationwide measure by applying weights to each inpatient discharge and its associated charge. Patient-level data are weighted with respect to the type of hospital where the service takes place. ${ }^{31}$ The nationwide discharge data are then summed with respect to each DRG.

American Hospital Association (AHA) Annual Survey. A data source commonly used in conjunction with the NIS is the AHA Annual Survey. The AHA has conducted the Annual Survey of hospitals since 1946 to construct a comprehensive health care provider database. Administered by Health Forum, the AHA Annual Survey contains hospital-specific data items on more than 6,500 US hospitals, including more than 1,000 data fields covering organizational structure, personnel, hospital facilities, services, and financial performance. ${ }^{32}$ One data element particularly beneficial in the creation of an output measure is the physical quantity measure of outpatient visits. Outpatient visits in community hospital data begins in 1988 and is available from AHA's web-based publication Chartbook.

This definition of the outpatient visit parallels that of the inpatient stay, because a patient's visit encompasses their course of medical service for one specific health issue. However, unlike an inpatient stay, a single person can record multiple outpatient visits on the same day, provided that each visit occurs at a different unit of the hospital.

National Hospital Ambulatory Medical Care Survey (NHAMCS). The NHAMCS provides a measure of outpatient visits separated into major

29. We are able to create time series of inpatient services by using DRG Versions 10, 18, and 24. The NIS provides yearly discharge data using these versions of the DRG system for time series analysis.

30. According to the NIS, government hospitals accounted for 21 percent of all United States hospitals in 2010.

31. These weights take into account geographic region, urban/rural location, teaching status, bed size, and ownership control; http://www.hcup-us.ahrq.gov/db/nation/nis/NIS_Introduction _2009.jsp.

32. American Hospital Association, AHA Annual Survey Database Fiscal Year 2011; http:// www.ahadataviewer.com/book-cd-products/AHA-Survey/. 
disease categories. ${ }^{33}$ The NHAMCS is produced by the Centers for Disease Control and Prevention's (CDC) National Center for Health Statistics (NCHS). It began in 1991 and is the principal federal source of information on the utilization of hospital emergency departments, outpatient departments, and hospital-based ambulatory surgery centers. ${ }^{34}$ It provides nationally representative estimates on the demographic characteristics of outpatients, diagnoses, diagnostic services, medication therapy, and the patterns of use of emergency and outpatient services in hospitals which differ in size, location, and ownership. ${ }^{35}$

The NHAMCS collects data from both hospital outpatient and emergency departments. An outpatient department is defined as a hospital facility where nonurgent ambulatory medical care is provided under the supervision of a physician. Outpatient clinics are included if ambulatory medical care is provided under the supervision of a physician and under the auspices of the hospital. Clinics where only ancillary services are provided or other settings in which physician services are not typically provided are not included. ${ }^{36}$

Emergency departments are sampled separately from outpatient departments. Statistics for each type of ambulatory care are calculated and published independently. Outpatient visits are the sum of both outpatient and emergency department visits.

As with the NIS data, we remove outpatient visits to government hospitals from the NHAMCS data set. The NIS includes emergency department patients who are admitted to the hospital, so we remove them from the NHAMCS to avoid double counting. While the number of outpatient visits is obtainable from the AHA Annual Survey and NHAMCS, neither of these sources provides cost or charge information.

CMS Statistical Supplement. The Medicare and Medicaid Statistical Supplement provides comprehensive annual data about Medicare, Medicaid, and other CMS programs. The supplement shows health expenditures for the entire US population, characteristics of the covered populations, use of services, and expenditures under these programs. ${ }^{37}$ The supplement includes

33. The scope of the AHA data set is greater than that of the NHAMCS. The AHA collects data from 98 percent of community hospitals, making it the preferred source of outpatient data.

34. Hospital-based ambulatory surgery centers were first added to this study in 2009 , and freestanding ambulatory surgery centers were added in 2010 .

35. Centers for Disease Control, Ambulatory Health Care Data, about the Ambulatory Health Care Surveys, National Hospital Ambulatory Medical Care Survey; http://www.cdc .gov/nchs/ahcd/about_ahcd.htm.

36. Centers for Disease Control, 2010 NHAMCS Micro-Data File Documentation; ftp:// ftp.cdc.gov/pub/Health_statistics/NCHs/Dataset_Documentation/NHAMCS/doc2010.pdf.

37. Centers for Medicare and Medicaid Services, Research, Statistics, Data and Systems, Medicare \& Medicaid Statistical Supplement; https://www.cms.gov/Research-Statistics-Data -and-Systems/Statistics-Trends-and-Reports/MedicareMedicaidStatSupp/index.html. 
a subsection for Hospital Outpatient Bills, Covered Charges, and Program Payments for various calendar years. ${ }^{38}$

Producer Price Index (PPI). Total revenue for each industry is deflated using the appropriate BLS PPI. ${ }^{39}$ The PPIs for general and specialty hospitals define output as a bundle of services provided for the treatment of a medical condition, not as the individual services rendered such as $\mathrm{x}$-rays, drugs, and medical supplies (Catron and Murphy 1996). The PPIs for both types of hospitals account for all services provided, including inpatient, outpatient, food services, and so forth, capturing the substitution effects of replacing costly inpatient treatments with more efficient outpatient procedures (Smith 2009). The general PPIs for each of the two types of hospitals measure actual payment to the hospital, not charges. Hospital bills are used to determine a price via the payments made by patients and third-party payers (Fixler and Ginsburg 2001).

BLS Industry Employment and Hours Data. Total annual hours are based primarily on data from the Current Employment Statistics (CES) survey, a monthly establishment survey conducted by BLS, and supplemented with data from the Current Population Survey (CPS), a monthly household survey conducted by the US Census Bureau for BLS. Employment and average weekly hours are measured separately for supervisory and nonsupervisory workers. The hours are treated as homogeneous and are directly aggregated. No adjustment was made to account for changes in labor composition.

\section{Appendix B}

\section{Using the Medical Expenditure Panel Survey (MEPS) to Construct an Outpatient Index}

The Medical Expenditure Panel Survey (MEPS) is an alternative data source that can be used to create an outpatient index in lieu of using CMS and NHAMCS data. This appendix will examine the MEPS and create an outpatient index using its data, while leaving the methodology unchanged from the original CMS and NHAMCS-based outpatient index.

Sponsored by the Agency for Healthcare Research and Quality (AHRQ), the MEPS is a series of nationally representative surveys of families and

38. Centers for Medicare and Medicaid Services, Research, Statistics, Data and Systems, Medicare \& Medicaid Statistical Supplement, 2011 Edition, Chapter 10: Medicare Hospital Outpatient Services, Table 10.4-Hospital Outpatient Bills, Covered Charges, and Program Payments Under Medicare, by Selected Reasons for the Visit: Calendar Year 2010; https://www.cms.gov/Research-Statistics-Data-and-Systems/Statistics-Trends-and-Reports/ MedicareMedicaidStatSupp/2011.html.

39. NAICS 6221 is deflated using PPI $622110622110 \mathrm{G}$, and NAICS 6223 is deflated using PPI $6223106223106 \mathrm{G}$. 
individuals, medical providers, and employers. The MEPS provides data on health services, health care utilization, and health expenditures in the United States. The Household Component (HC) of the MEPS surveys approximately 15,000 households each year and collects detailed information for each person in the household on health service type, frequency of use, and health conditions, as well as data on health insurance. In the Medical Provider Component (MPC), a sample of medical providers are contacted to acquire data that household respondents may not be able to accurately supply, such as information on visit dates, diagnosis and procedure codes, charges, and payments. The MEPS data are available starting in 1996 and can be analyzed at either the person or event level and weights are provided to produce national estimates. The number of outpatient visits and expenditures are reported on an ICD-9-CM basis.

As with the outpatient measure used in the main body of this chapter, the American Hospital Association total outpatient visits for each year is used as the baseline for the MEPS outpatient index. Detailed ICD-9-CM visits from the MEPS are aggregated to the major disease category level and are used to disaggregate the quantity of total visits as reported by the AHA. The ICD-9-CM charge data from the MEPS is also aggregated to the major disease category level and combined with major disease category-level visits to create an outpatient index. The MEPS charge values and the proportions of MEPS visits for each major disease category to total MEPS visits are held constant from 1993 to 1996. The following equation is used to create the outpatient index based on MEPS data. Equation (B.1):

$$
\frac{Q_{t}}{Q_{t-1}}=\exp \left[\sum_{i=1}^{n} w_{i, t}\left(\ln \left(\frac{q_{i, t}}{q_{i, t-1}}\right)\right)\right]
$$

where

$Q_{t} / Q_{t-1}=$ the ratio of outpatient output in the current year $(t)$ to the previous year $(t-1)$,

$n=$ the number of major disease categories

$\ln \left(q_{i, t} / q_{i, t-1}\right)=$ the natural logarithm of the ratio of the quantity of outpatient visits for major disease category $i$ in the current year to the quantity in the previous year, and $w_{i, t}=$ the average value share weight for major disease category $i$.

\section{Critique of the MEPS}

While the CMS and NHAMCS-based outpatient index relies upon visit and charge data from separate sources, the MEPS-based index uses a single source for this data. This provides a level of consistency not found in the original outpatient measure. In addition, while CMS data account for only Medicare and Medicaid populations, the MEPS does not focus on any subset of the US population as it is a nationally representative survey of the US 


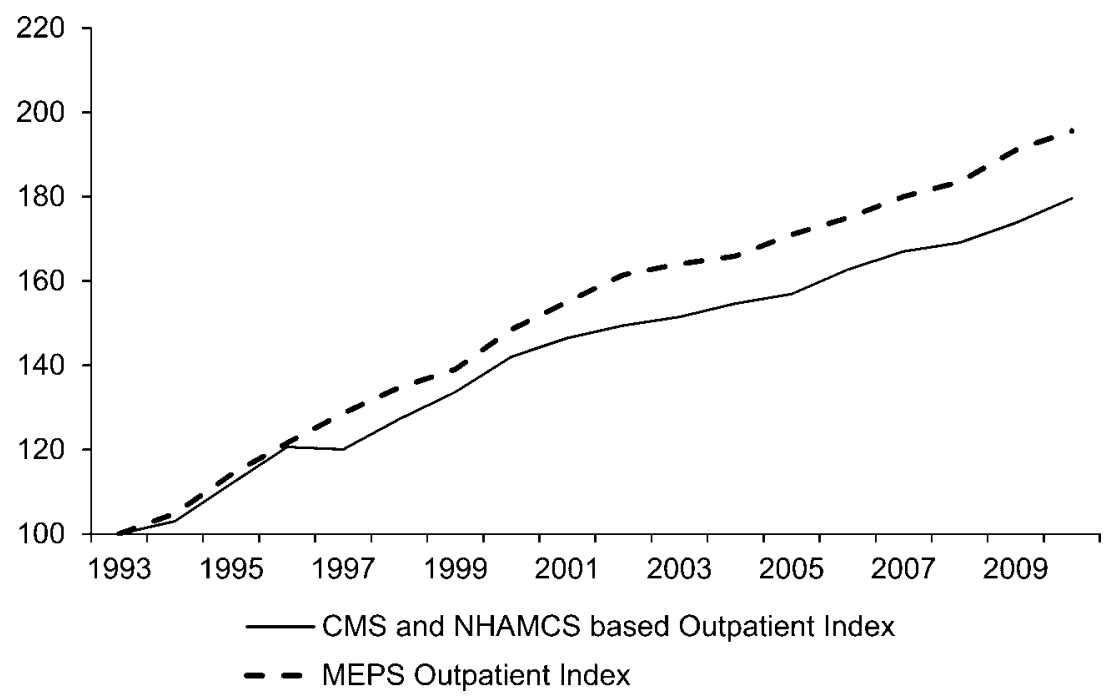

Fig. 5B.1 Index of outpatient services using MEPS 1993-2010

civilian noninstitutionalized population. Emergency room visit data from the MEPS are available; however, these figures have not been incorporated into this measure. Further research into this topic has the potential to augment the outpatient index.

The MEPS definition of an outpatient department encompasses not only outpatient centers within hospitals, but also those that are affiliated with hospitals. $^{40}$ The hospital-affiliated outpatient departments may cross NAICS industry borders, creating inconsistency with the labor input measure. Additionally, each MEPS outpatient visit has up to four condition codes that are sequenced in the data files in the order reported by the household respondent and not in order of importance or severity. ${ }^{41}$ The lack of condition-specific charge data for respondents with multiple condition codes prevents accounting for multiple condition codes in the MEPS measure.

\section{Results}

The outpatient services index for community hospitals created with MEPS data shows positive growth for 1993-2010. As figure 5B.1 demonstrates, the

40. The AHRQ, MEPS, Medical Expenditure Panel Survey Household Component Main Study, Glossary, Outpatient Department; http://meps.ahrq.gov/survey_comp/hc_ques_glossary .shtml.

41. The AHRQ, MEPS, MEPS HC-135F: 2010 Outpatient Department Visits, 2.5.4 Conditions and Procedures Codes; http://meps.ahrq.gov/mepsweb/data_stats/download_data/pufs/ h135f/h135fdoc.shtml. 
output index is closely aligned with the output index based on CMS charges and NHAMCS visits.

The positive trend of both outpatient measures shows that using either the MEPS data or the combination of CMS and NHAMCS data are both viable options. The choice of data source for the outpatient index thus depends on which survey is more reliable and how well it aligns with the AHA and BLS definitions of community hospitals.

\section{Appendix C}

\section{Adjusting the Inpatient Index for Survival Rates}

The NIS data set that is used to create the inpatient index includes information regarding whether the patient was discharged alive or dead. From this information, we can calculate survival rates for each year and each DRG. The survival rate is defined as the number of patients who were discharged alive divided by the total number of patients.

It has been suggested that incorporating the year-to-year change in survival rates into the output index may be an indirect way to measure the quality change in hospital treatments. For each DRG, yearly survival rates are calculated. The change in survival rate from year $t-1$ to year $t$ is then multiplied by the number of discharges in year $t$. In effect, the quantity of discharges for each year is adjusted upward for gains in the survival rate or downward for decreases in the survival rate.

The effects of this adjustment are surprisingly negligent. Figure 5C.1 shows both the survival-adjusted inpatient index and the unadjusted index. The two lines are virtually indistinguishable.

For most years and most DRGs, the change in survival rates is very small. There are also a significant number of DRGs where the survival rate remains unchanged or decreases. Table 5C.1 shows the ratio of survival rate changes by year.

In most (but not all) years, the number of DRGs where the survival rate increased outnumbered those where the survival rate decreased. However, the trend is not so overwhelming so as to actually affect the output index in a significant manner.

There are other possible aspects that could be included in creating a survival-adjusted inpatient measure. For example, the adjustment could be made only upon those DRGs that have a high rate of mortality in the first place ("high" being defined in any number of ways). The concept in this case is that some DRGs have inherently very low mortality rates. In those cases where patients do die, the hospital was likely not responsible, and thus any change in mortality rates for these DRGs are not indicative of hospital quality. 


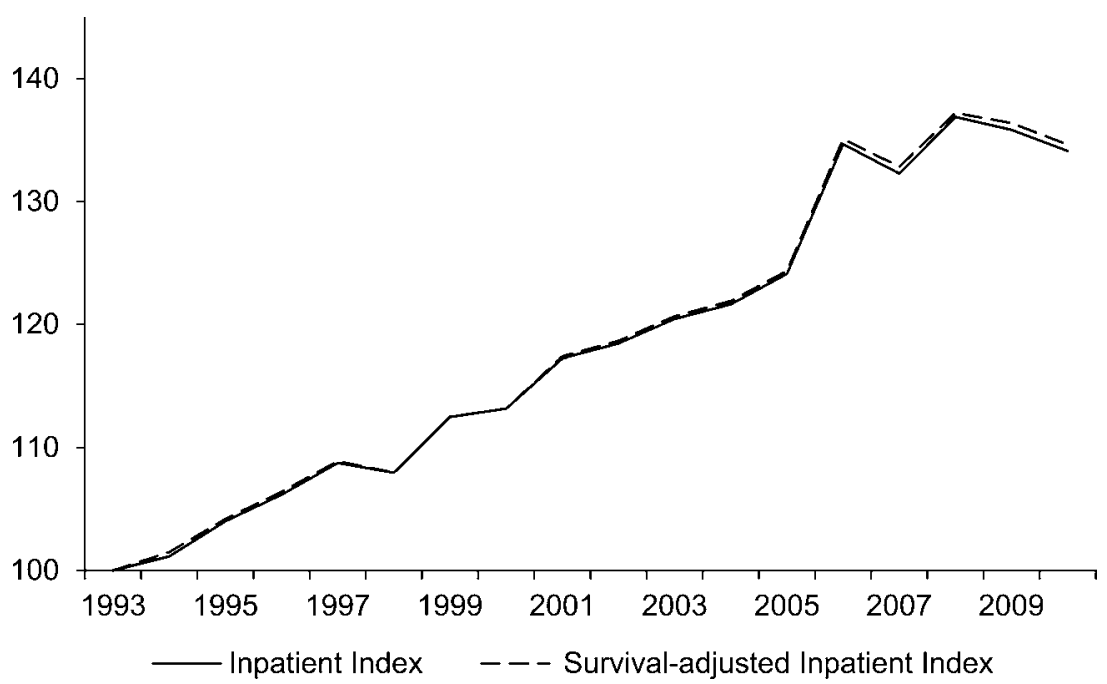

Fig. 5C.1 Survival-adjusted inpatient index

Table 5C.1 Percent of DRGs where the survival rate

\begin{tabular}{cccc}
\hline Year & $\begin{array}{c}\text { Increased } \\
(\%)\end{array}$ & $\begin{array}{c}\text { Decreased } \\
(\%)\end{array}$ & $\begin{array}{c}\text { Stayed the same } \\
(\%)\end{array}$ \\
\hline 1994 & 59 & 31 & 10 \\
1995 & 54 & 34 & 12 \\
1996 & 51 & 35 & 14 \\
1997 & 52 & 35 & 14 \\
1998 & 39 & 49 & 12 \\
1999 & 38 & 50 & 12 \\
2000 & 47 & 41 & 12 \\
2001 & 47 & 40 & 12 \\
2002 & 52 & 35 & 13 \\
2003 & 50 & 35 & 15 \\
2004 & 49 & 35 & 15 \\
2005 & 50 & 35 & 15 \\
2006 & 46 & 38 & 16 \\
2007 & 52 & 32 & 16 \\
2008 & 41 & 45 & 14 \\
2009 & 51 & 34 & 15 \\
2010 & 50 & 33 & 17 \\
\hline
\end{tabular}

The question of how to link survival rates with actual hospital actions is crucial. It only makes sense to include a survival adjustment if the change in survival rates is directly attributable to the quality of hospital services. But mortality rates can be affected by numerous outside forces, such as public health trends, the patient's adherence to treatment, and random chance. A 
subtle factor that influences survival rates are choices made by gravely ill patients. If a patient chooses risky treatment in a hospital versus palliative care in a hospice, that influences the hospital's survival rate.

At this time, it is not practical to incorporate a survival adjustment into the inpatient index. However, as a potential method for incorporating quality data into a physical quantity output measure, this type of adjustment may be worth further study.

\section{References}

Aizcorbe, Ana, and Nicole Nestoriak. 2008. "The Importance of Pricing the Bundle of Treatments." Working Paper, Bureau of Economic Analysis, July.

- 2011. "Changing Mix of Medical Care Services: Stylized Facts and Implications for Price Indexes.” Journal of Health Economics 30 (3): 568-74.

Catron, Brian, and Bonnie Murphy. 1996. "Hospital Price Inflation: What Does the New PPI Tell Us?” Monthly Labor Review July:24-31.

Cylus, Jonathan, and Bridget Dickensheets. 2007. "Hospital Multifactor Productivity: A Presentation and Analysis of Two Methodologies." Health Care Financing Review 29 (2): 49-64.

Douglas, James. 2006. "Measurement of Public Sector Output and Productivity." New Zealand Treasury Policy Perspectives Paper, November.

Fixler, Dennis, and Mitchell Ginsburg. 2001. "Health Care Output and Prices in the Producer Price Index." Medical Care Output and Productivity January:221-70.

Henderson, Richard. 2012. "Industry Employment and Output Projections to 2020." Monthly Labor Review January:65-83.

Hospital Quality Valuation Team. 2008. "Proposal for Adjusting the General Hospital Producer Price Index for Quality Change.” Paper presented at the National Bureau of Economic Research Summer Institute Conference on Research in Income and Wealth, July 14.

Lane, Sarah Gunther. 2001. A Community Leader's Guide to Hospital Finance: Evaluating How a Hospital Gets and Spends its Money. Boston: The Access Project.

National Research Council. 2010. "Accounting for Health and Health Care: Approaches to Measuring the Sources and Costs of their Improvement." Panel to Advance a Research Program on the Design of National Health Accounts, Committee on National Statistics.

Rosen, Allison B., and David M. Cutler. 2007. "Measuring Medical Care Productivity: A Proposal for US National Health Accounts." Survey of Current Business June:54-88.

Smith, Shelly. 2009. "A New Approach to Price Measures for Health Care." Survey of Current Business February:17-20.

Stranges, Elizabeth, C. Allison Russo, and Bernard Friedman. 2009. "Procedures with the Most Rapidly Increasing Hospital Costs, 2004-2007." HCUP Statistical Brief no. 82, Healthcare Cost and Utilization Project, December.

Statistics New Zealand. 2013. "Education and Health Industry Productivity 19962011." http://www.stats.govt.nz/.

Triplett, Jack. 2012. "Health System Productivity." Oxford Handbook of Health Economics, edited by Sherry Glied and Peter C. Smith. DOI: 10.1093/oxfordhb/ 9780199238828.013.0030. 